\title{
Mapping ultrafast ionization of atoms and clusters with terahertz-streaking delay
}

\author{
Tim Oelze, ${ }^{1}$ Bernd Schütte, ${ }^{2}$ Jan P. Müller, ${ }^{3}$ Maria Müller, ${ }^{3}$ Marek Wieland, ${ }^{4,5}$ Ulrike Frühling, ${ }^{4,5}$ Markus Drescher,,${ }^{4,5}$ \\ Torsten Golz, ${ }^{6}$ Alaa Al-Shemmary, ${ }^{6}$ Nikola Stojanovic, ${ }^{6}$ and Maria Krikunova ${ }^{1,7, *}$ \\ ${ }^{1}$ Institut für Optik und Atomare Physik, Technische Universität Berlin, Strasse des 17. Juni 135, ER 1-1, 10623 Berlin, Germany \\ ${ }^{2}$ Max-Born-Institut, Max-Born-Straße 2a, 12489 Berlin, Germany \\ ${ }^{3}$ Institut für Optik und Atomare Physik, Technische Universität Berlin, Hardenbergstr. 36, EW 3-1, 10623 Berlin, Germany \\ ${ }^{4}$ Institut für Experimentalphysik, Universität Hamburg, Luruper Chaussee 149, 22761 Hamburg, Germany \\ ${ }^{5}$ Center for Ultrafast Imaging, Luruper Chaussee 149, 22761 Hamburg, Germany \\ ${ }^{6}$ Deutsches Elektronen-Synchrotron DESY, Notkestraße, 22603 Hamburg, Germany \\ ${ }^{7}$ ELI Beamlines, Institute of Physics, Czech Academy of Science, Na Slovance 2, 18221 Prague, Czech Republic
}

(Received 12 December 2018; published 24 April 2019)

\begin{abstract}
We apply THz-field streaking to temporally resolve the ultrafast ionization of neutral cluster vs atomic targets exposed to intense ultrashort soft x-ray pulses from a free-electron laser (FEL). In the experiment pristine Xe and mixed $\mathrm{Xe} / \mathrm{Ar}$ clusters as well as Xe atoms are ionized in the vicinity of the $4 d \rightarrow \varepsilon f$ giant resonance of $\mathrm{Xe}$. We compare the relative streaking delay between the center-of-mass oscillations of electrons that have final kinetic energies in the spectral region of the $\mathrm{Xe}(4 d)$ photoline. Our results show that clusters are ionized at the beginning of the $100 \mathrm{fs}$ FEL pulse as supported by calculations of target frustration in the focal volume. We have identified a significantly larger 40 fs relative streaking delay between mixed Xe-Ar clusters vs Xe atoms compared to a 15 fs relative delay found for pristine Xe clusters. This is attributed to the high sensitivity of our spectroscopic measurement to the degree of condensation of the cluster target. Our results show that $\mathrm{THz}$ streaking is a powerful technique to temporally study electron emission from extended targets under intense FEL radiation on time scales that are significantly shorter than the FEL pulse duration.
\end{abstract}

DOI: 10.1103/PhysRevA.99.043423

\section{INTRODUCTION}

The remarkable development of short-wavelength freeelectron lasers (FELs) as well as laboratory-scale sources based on high harmonic generation have opened up a new field of ultrafast $\mathrm{x}$-ray physics with unique opportunities for the studies of structure and dynamics of matter on their natural time and length scales [1-3]. High irradiation intensity combined with short pulse duration provided access to a new parameter regime of nonlinear x-ray physics [4-7] and has led to the observation of novel phenomena such as $\mathrm{X}$-ray induced transparency [8], collective plasmalike states [9], or interatomic Coulombic decay cascades [10]. Ultrafast time-resolved spectroscopy using FEL pulses is now able to trace the dynamics of electronic and nuclear rearrangement in molecules [11,12]. Another prominent example is the serial time-resolved crystallography that has provided unprecedented insights into the conformational changes triggered by the excitation of Photosystem II with visible light [13].

For many applications, the understanding of the electron dynamics of a system exposed to ultrashort and ultraintense FEL pulses is essential. When a nanoscale system is hit by an intense FEL pulse, complex electron and nuclear dynamics evolve on time scales from a few femtoseconds to several hundreds of picoseconds [14-16]. While the processes evolving in the nanoscale system after the FEL pulse has

\footnotetext{
*maria.krikunova@physik.tu-berlin.de
}

ended are accessible in the time domain by one- or twocolor pump-probe techniques [17-22], time-resolved studies covering time scales shorter than the FEL pulse duration remain challenging [23]. A technique capable of accessing these time scales is light-field streaking that is sensitive to the time instance at which an electron is freed into the continuum [24-26]. This sensitivity allows one to resolve the electron dynamics within the XUV pulse [24,27,28]. Lightfield streaking was applied to map the time difference between electrons emitted from two different orbitals of the same atom [27] or from localized vs delocalized states of a solid sample [28] with attosecond time resolution. Recently this technique has been applied to study electron scattering in dielectric nanoparticles [29]. In the current study we use the streaking delay [27-29] to resolve how fast neutral clusters become ionized by an intense FEL pulse.

\section{EXPERIMENT}

The principle of our experimental approach is presented in Fig. 1. Figures 1(a) and 1(b) (contour plot) show the electron kinetic-energy distributions vs electron birth time, as calculated for $\mathrm{Ar}_{147}$ clusters using a quasiclassical molecular dynamics approach [30]. Three distinct signatures in the electron kinetic-energy spectrum have been studied previously [31,32]. (i) The spectral region around the atomic photoline corresponds to electron emission from neutral clusters [blue shaded area in Figs. 1(a) and 1(b) marks the $\operatorname{Ar}(3 p)$ photoline]. (ii) A plateau region that is formed of electrons emitted from 


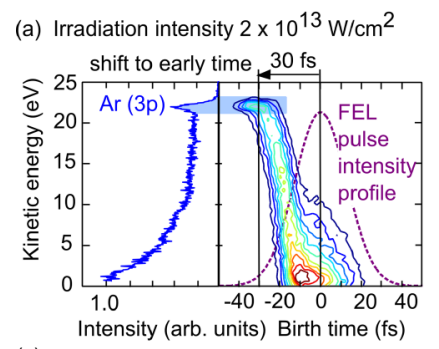

(b) Irradiation intensity $5 \times 10^{11} \mathrm{~W} / \mathrm{cm}^{2}$

(c)
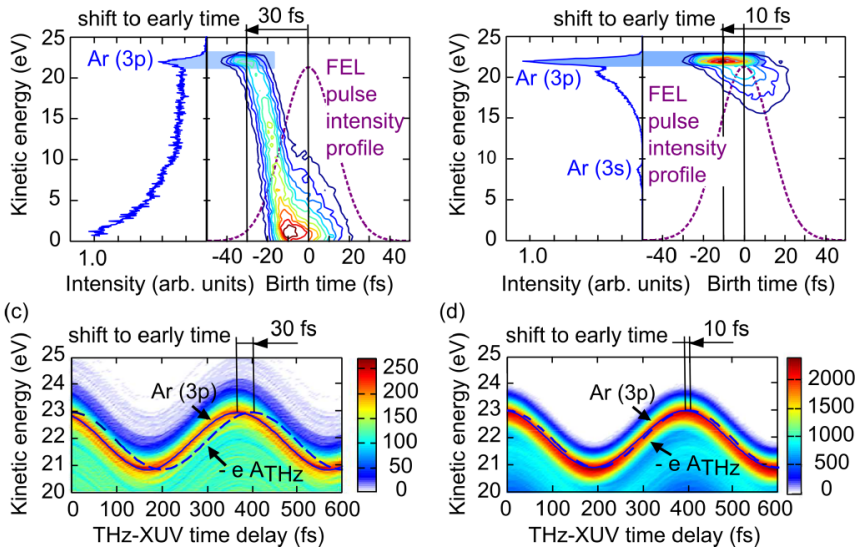

FIG. 1. Principle of the THz streaking experiment. (a),(b) Results of molecular dynamics simulations (reproduced from Ref. [30]) showing electron kinetic-energy distribution vs electron birth time (contour plot) and electron spectrum (left panel) for two different XUV intensities. The simulation was performed for $\mathrm{Ar}_{147}$ clusters exposed to a $30 \mathrm{fs}$ FWHM FEL pulse (violet dashed line) of $38 \mathrm{eV}$ photon energy. (c),(d) Streaking spectrograms simulated for corresponding time-energy distributions.

already ionized clusters. These electrons are energetically downshifted due to the growing electrostatic potential of the cluster. (iii) An energy tail with maximum yield at zero kinetic energy. This part of the spectrum is formed by electrons that are initially trapped by the cluster potential and that are emitted from the expanding cluster at a later stage. Note the shift of the electron emission from the spectral region of the $\operatorname{Ar}(3 p)$ line (the blue shaded area) towards early time with respect to the FEL pulse maximum. For higher irradiation intensity the shift becomes larger [compare Figs. 1(a) and 1(b)]. According to our calculations of corresponding streaking spectrograms $[25,26]$ the shift can be accessed in the time domain [Figs. 1(c) and $1(\mathrm{~d})]$ :

$$
p_{\text {final }}(\tau, t)=p_{\text {in }}(t)-e A_{\max } \cos \left[\omega(t-\tau)-\varphi_{0}\right],
$$

where $p_{\text {in }}$ and $p_{\text {final }}$ are the initial and final distributions of the electron momenta (we consider electrons that are emitted along the polarization direction of the laser dressing field), $e$ is the electron charge, $A_{\max }$ is the amplitude of the vector potential, $\omega$ is the angular frequency of the dressing field, $t$ describes the time axis of the ionizing pulse (with the time zero set to the pulse maximum), $\tau$ is the relative time delay between the ionizing pulse and the dressing field, and $\varphi_{0}$ is the initial phase of the dressing field. Since the dynamics takes place on a femtosecond time scale, a dressing field in the terahertz $(\mathrm{THz})$ frequency region is used $[33,34]$. In the calculation we use $A_{\max }=4 \times 10^{-7} \mathrm{~V} \mathrm{~s} / \mathrm{m}$ corresponding to a maximal electric-field amplitude of $6 \mathrm{MV} / \mathrm{m}, \omega=2 \pi / \mathrm{T}$ with an oscillation period of $T=400 \mathrm{fs}$, and $\varphi_{0}=\pi$.

$\operatorname{The} \operatorname{Ar}(3 p)$ line in the calculated streaking spectrograms [Figs. 1(c) and 1(d)] shows a characteristic oscillatory behavior that follows the oscillation of the vector potential $A_{T H z}=$ $A_{\max } \cos \left[\omega(t-\tau)-\varphi_{0}\right]$ (blue dashed line). In this plot $A_{T H z}$ is scaled to the kinetic-energy shift $\triangle W$ according to

$$
\Delta W_{\|} \approx-\sqrt{\frac{2 W_{0}}{m_{e}}} e A_{T H z},
$$

where $m_{e}$ is the electron mass and $W_{0}$ is the kinetic energy of the electron without the laser dressing field. In streaking spectrograms the shift of $\operatorname{Ar}(3 p)$ emission from neutral clusters towards the leading edge of the FEL pulse appears as a shift of the $\operatorname{Ar}(3 p)$ center-of-mass (COM) oscillation with respect to the oscillation of $-e A_{T H z}$ [Figs. 1(c) and 1(d), solid and dashed lines].

In order to resolve the ionization of neutral clusters vs atoms in our experiment, we chose pristine $\mathrm{Xe}$ and mixed $\mathrm{Xe} / \mathrm{Ar}$ clusters. The FEL photon energy was in the vicinity of the $4 d \rightarrow \varepsilon f$ giant resonance of $\mathrm{Xe}$ [35]. We note that the experimental parameters used in our study differ from parameters used in the simulation (Fig. 1). This, however, does not change the physical mechanism that is responsible for the ionization of clusters [36]. The experiment was performed at BL 3 of the Free-Electron Laser facility in Hamburg (FLASH), which provides intrinsically synchronized XUV and multicycle $\mathrm{THz}$ pulses [33,37]. At the experimental end station the XUV FEL pulses at $13.5 \mathrm{~nm}$ wavelength $(92 \mathrm{eV}$ photon energy) were colinearly overlapped with $\mathrm{THz}$ pulses of $2.5 \mathrm{THz}$ central frequency (120 $\mu \mathrm{m}$ wavelength). A Mo/Si multilayer mirror (focal length $f=2100 \mathrm{~mm}$ ) was used to adjust the XUV and THz beamline lengths and to focus the XUV beam. The XUV peak irradiation intensity in the interaction region was about $9 \times 10^{13} \mathrm{~W} / \mathrm{cm}^{2}$ by assuming a focal spot size of about $30 \mu \mathrm{m}$ full width at half maximum (FWHM), $100 \mu \mathrm{J}$ average FEL pulse energy, and $100 \mathrm{fs}$ (FWHM) pulse duration (estimated from parameters of the FEL operation). An off-axis parabolic mirror $(f=320 \mathrm{~mm})$ was employed to focus the $\mathrm{THz}$ beam, leading to a focused spot size of $1.8 \mathrm{~mm}$ (FWHM). The maximal THz electric-field strength was $6 \mathrm{MV} / \mathrm{m}$ as estimated from streaking spectrograms. With an estimated Rayleigh length of $30 \mathrm{~mm}$ and a target length of about $1.5 \mathrm{~mm}$ the estimated Gouy phase shift [38] of the $\mathrm{THz}$ field that introduces a change of the XUV-THz delay over the interaction region was about 5 fs. A delay stage available in the $\mathrm{THz}$ brunch was used to vary the relative time delay of the $\mathrm{THz}$ pulses with respect to the XUV pulses in steps of 30 fs. A time-of-flight spectrometer [33,39] was used to record photoelectron spectra parallel to the polarization of the $\mathrm{THz}$ field. Electron spectra were averaged over about 100 single FEL shots at each $\mathrm{THz}-\mathrm{XUV}$ time delay and arranged into streaking spectrograms.

Clusters were produced at room temperature by an adiabatic expansion of pure Xe gas or a gas premix of $2 \% \mathrm{Xe}$ in $\mathrm{Ar}$ through a pulsed $100 \mu \mathrm{m}$ conically shaped nozzle with a halfopening angle of $15^{\circ}$. The cluster jet was sent through two skimmer systems to reduce the contribution of uncondensed gas. The pressure within the experimental chamber was maintained at $5 \times 10^{-7}$ mbar. Additionally a piezodriven slit was installed close to the interaction region to further restrict the cluster beam. The stagnation pressure behind the nozzle was varied between 6 and 22.7 bar in order to produce Xe clusters with an average number of atoms $\langle N\rangle$ between 5700 and 130000 as estimated by scaling laws [40]. Expansion of 

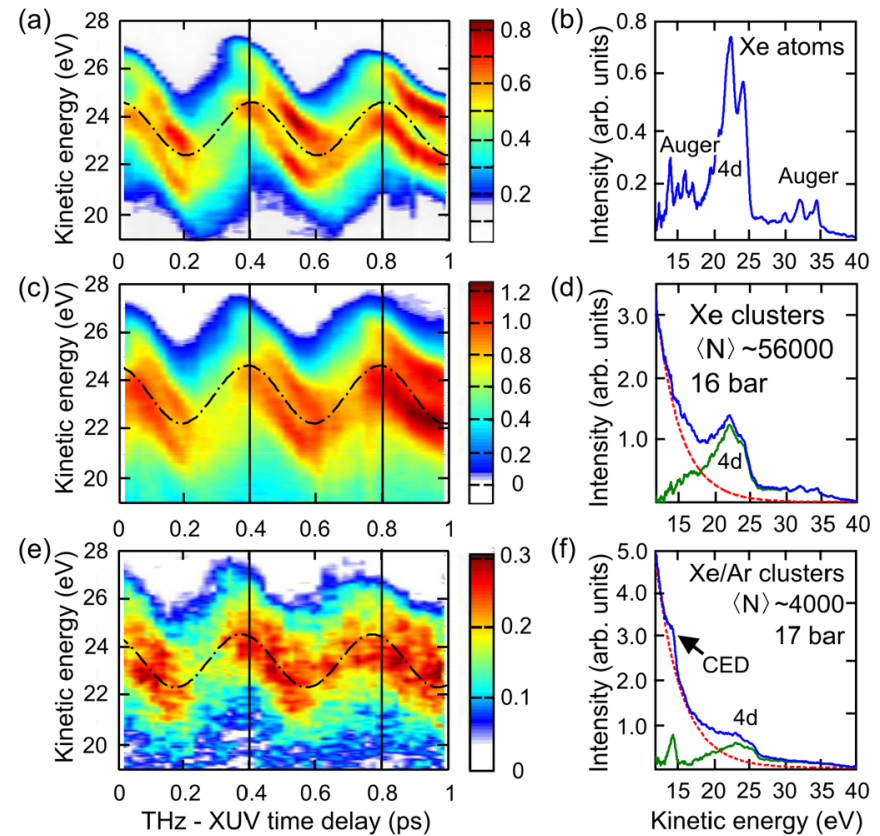

FIG. 2. THz streaking spectrograms (left panels) and corresponding THz-field free-electron kinetic-energy spectra (right panels) recorded in atomic (a),(b), pristine Xe cluster (c),(d), and mixed $\mathrm{Xe} / \mathrm{Ar}$ (e),(f) cluster targets. The dash-dotted lines in streaking spectrograms correspond to the center-of-mass approximations of the $\mathrm{Xe}(4 d)$ spectral region.

the $\mathrm{Xe}$-Ar gas premix at 17 bar stagnation pressure resulted in the generation of mixed $\mathrm{Xe} / \mathrm{Ar}$ clusters with an average number of atoms $\langle N\rangle \approx 4000$ [41,42]. According to previous investigations this results in mixed $\mathrm{Xe} / \mathrm{Ar}$ clusters with about $800 \mathrm{Xe}$ atoms preferably located in the core and a few layers of Ar atoms on top of it [41-43].

\section{RESULTS AND DISCUSSION}

Figure 2 shows streaking spectrograms (left panels) and the corresponding THz-field free-electron kinetic-energy spectra (right panels) measured upon ionization of Xe atoms (a),(b), pristine Xe clusters (c),(d), and Xe/Ar clusters (e),(f). All spectrograms were measured within the same delay range. Due to the frequency chirp of the FEL pulse [33] the spectrum is broader on the rising slope of the vector potential $-e A_{T H z}$ and narrower on the falling slope. Additional changes of the signal level might be caused by changes of the average FEL pulse energy during the scan time $(11 \mathrm{~min})$. The cluster electron spectra are similar to the simulated spectrum in Fig. 1(a) and show the spectral contributions of the atomic photoline $[\mathrm{Xe}(4 d)$ electrons] and a plateau region as well as a contribution from a nanoplasma with a maximum yield at zero kinetic energy [30-32,44,45]. In Xe/Ar clusters the signature at $\sim 13.7 \mathrm{eV}$ [Fig. 2(f)] is due to correlated electronic decay taking place during the cluster expansion [39]. In order to increase the visibility of the $\mathrm{Xe}(4 d)$ spectral region the nanoplasma contribution was fitted with an exponential function [red dashed line in (d) and (f)] and subtracted [green line in (d) and (f)] in the cluster spectrograms. The dashdotted lines (Fig. 2, left panels) map the COM oscillation
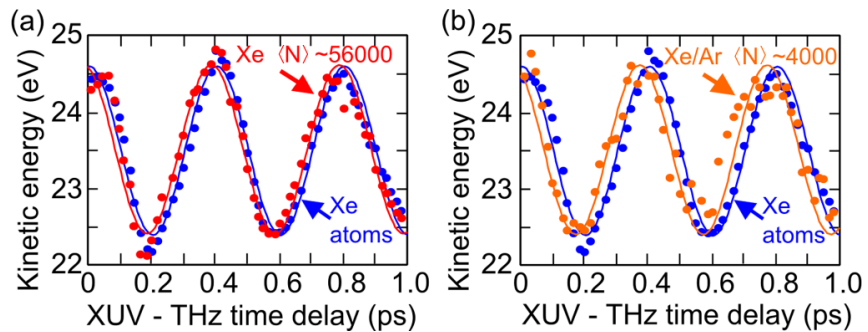

FIG. 3. Center-of-mass values (circles) and the corresponding approximations (solid lines) of the $\mathrm{Xe}(4 d)$ spectral region for atoms vs pristine Xe clusters (a) and for atoms vs Xe/Ar clusters (b). Data are extracted from the corresponding THz-streaking spectrograms of Fig. 2.

of the $\mathrm{Xe}(4 d)$ spectral region. The following procedure has been used to approximate the COM values. (i) The region of interest is selected between 21 and $26 \mathrm{eV}$ to cover the oscillation of the $\mathrm{Xe}(4 d)$ spectral region. At each time delay of a streaking spectrogram the COM value is calculated within a defined region of interest $(21-26 \mathrm{eV})$ in the electron spectrum. Figure 3 shows COM values (circles) calculated from corresponding atomic and cluster streaking spectrograms of Fig. 2 (left panels). (ii) The COM data are approximated by Eqs. (1) and (2) to describe the THz-XUV time-delay dependent kinetic-energy shift $\Delta W$ (solid lines in Fig. 3). To find the phase of the cosine term in Eq. (1) we perform a Fourier analysis of COM data and extract the phase of the Fourier component at the central frequency of the THz streaking field. The estimated uncertainty in the approximation of the COM data with the cosine function is $\sim 6$ fs.

A relative shift of the COM oscillation from clusters vs atomic targets towards early THz-XUV time delays is visible in Fig. 3. This demonstrates a faster ionization of a neutral cluster target with respect to atoms as expected from calculations in Fig. 1. Notably, within the studied cluster size range the average shift of the $\mathrm{COM} \mathrm{Xe}(4 d)$ oscillation between Xe atoms and Xe clusters was the same, namely, 14 fs for $\langle N\rangle \sim 5700$ and 11 fs for $\langle N\rangle \sim 56000$, as well as 14 fs and 16 fs obtained in two consecutive measurements on $\langle N\rangle \sim 130000$ clusters. From two consecutive measurements on mixed $\mathrm{Xe} / \mathrm{Ar}$ clusters composed of $\langle N\rangle_{X e} \sim 800$ and $\langle N\rangle_{A r} \sim 3200$ the extracted COM shift was larger, namely, 40 fs and $38 \mathrm{fs}$, respectively.

We explain the shorter streaking delay found in pristine $\mathrm{Xe}$ clusters by the presence of the uncondensed Xe gas in the cluster target. Indeed, the gas mixture used to produce $\mathrm{Xe} / \mathrm{Ar}$ clusters consists of only $2 \% \mathrm{Xe}$. According to previous investigations the $\mathrm{Xe}$ enrichment factor in mixed clusters is $\sim 10$ [41-43]. By the coexpansion of the $\mathrm{Xe} / \mathrm{Ar}$ gas premix $\mathrm{Xe}$ atoms nucleate in the core. Ar atoms act as a seeding gas and partially evaporate off from the surface of a growing $\mathrm{Xe}$ cluster $[41,43]$. Therefore, the contribution of $\mathrm{Xe}(4 d)$ electrons from uncondensed gas is substantially eliminated in the Xe/Ar cluster target. This finding is attributed to the high sensitivity of the spectroscopic measurements to the degree of the target condensation.

To understand the insensitivity of the streaking delay to the cluster size we have modeled the ionization of neutral targets 

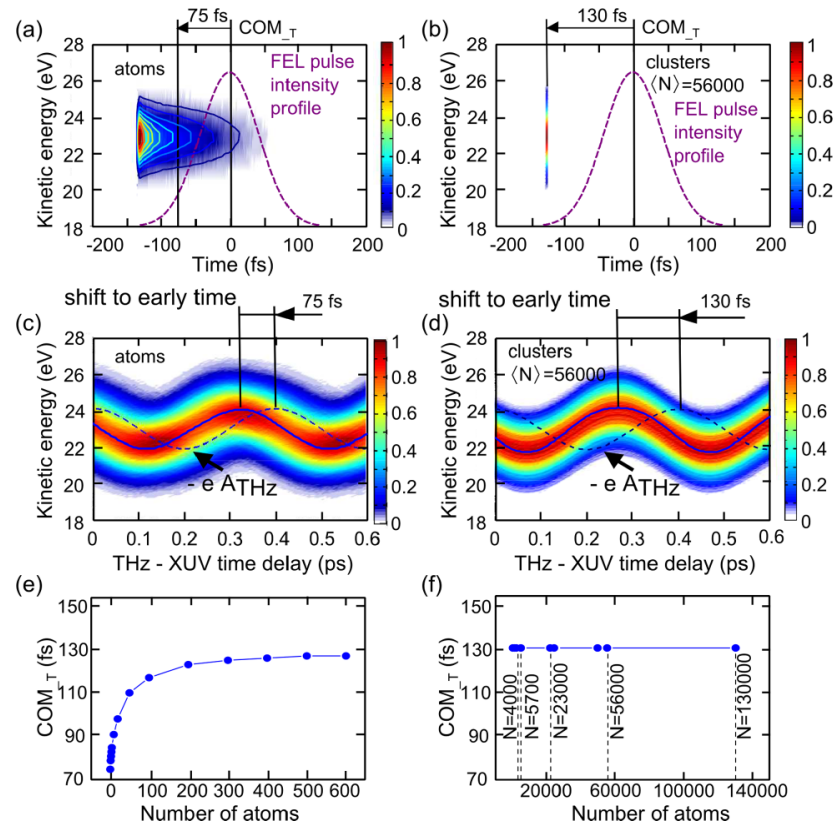

FIG. 4. Model calculations of the ionization of neutral targets in the focal volume. Calculated time-energy distributions of the electron emission for atomic (a) and cluster (b) targets. $\mathrm{COM}_{T}$ marks the center-of-mass coordinate of the temporal emission profile. It is calculated on the time axis of the FEL pulse as a reference frame. Streaking spectrograms calculated for corresponding atomic (c) and cluster (d) time-energy distributions. (e),(f) The center-of-mass coordinate $\mathrm{COM}_{T}$ calculated for the target with increasing number of atoms. Cluster sizes investigated in the current study are marked by dashed lines.

in the focal volume. Ionization was calculated numerically by considering a one-photon ionization rate:

$$
\Gamma=N_{\text {atoms }} \sigma \Phi,
$$

where $N_{\text {atoms }}$ is the number of atoms in the system, $\sigma=25$ Mbarn is the absorption cross section of Xe [35], and $\Phi=$ $I / \hbar \omega$ is the photon flux density. In the interaction region we have assumed a Gaussian radial and temporal intensity distribution with the peak intensity of $I_{0}=9 \times 10^{13} \mathrm{~W} / \mathrm{cm}^{2}$. The sampling interval of the pulse pedestal has been restricted to $\pm 130 \mathrm{fs}$ on the time axis and to $75 \mu \mathrm{m}$ for the radial profile. The intensity dependence along the FEL beam propagation axis was neglected due to the long $(\sim 2.5 \mathrm{~mm})$ Rayleigh length. Figure 4 shows calculated time-energy distribution for the electron emission from atoms (a) and clusters (b) with $\langle N\rangle=56000$. We have assumed a Gaussian profile for the electron kinetic-energy distribution [vertical axis in Figs. 4(a) and 4(b)]. In contrast to the full molecular dynamics simulation shown in Figs. 1(a) and 1(b) only the emission of a first electron from neutral clusters has been considered in Fig. 4(b). Our calculations show a strong ionization of atomic target already in the rising slope of the FEL pulse. Note that the one-photon ionization rate of clusters [Eq. (3)] is substantially higher due to the large number of atoms $N_{\text {atoms. }}$. This results in a much faster ionization of clusters compared to atoms.

The electron streaking [Figs. 4(c) and 4(d)] tracks the coordinate of the COM of the electron emission on the time axis of the FEL pulse. We refer to it as $\mathrm{COM}_{T}$ (where $T$ stands for the FEL time axis) in order to distinguish it from the COM shift of the $\mathrm{Xe}(4 d)$ photoline oscillation we have referred to so far. In the experiment the time axis of the FEL pulse is unknown; therefore, only the relative difference between $\mathrm{COM}_{T}$ coordinates in clusters vs atoms can be compared. We have identified $\mathrm{COM}_{T}=75$ fs for atoms and $\mathrm{COM}_{T}=$ 130 fs for clusters of $\langle N\rangle=56000$, resulting in a 55 fs relative streaking delay between atoms and clusters. This result is within the reasonable agreement with the 40 fs extracted in the experiment for Xe/Ar mixed clusters. Deviations can be explained mainly by shot-to-shot fluctuations of the temporal and spatial intensity distributions of FEL pulses and, additionally, by the presence of residual uncondensed gas as well as by electrons emitted from ionized clusters that have kinetic energies between 21 and $26 \mathrm{eV}$. Figures 4(e) and 4(f) show the $\mathrm{COM}_{T}$ coordinate calculated as a function of the number of atoms in the system. Note that the $\mathrm{COM}_{T}$ increases very fast [Fig. 4(e)] and reaches a saturation value for $N \sim 500$ [Fig. 4(f)]. Due to the fast ionization of clusters with $N>500$ the streaking delay is insensitive to the studied cluster size [see Fig. 4(f), dashed lines].

\section{SUMMARY}

We have exploited the sensitivity of the light field streaking technique to the instance of the electron emission to resolve how fast neutral clusters with respect to atoms become ionized under intense FEL radiation. The ultrafast ionization of a target in the focus volume is crucial for the understanding of multiphoton and nonlinear x-ray physics phenomena [4-10]. The delay in streaking spectrograms reveals ultrafast ionization of neutral clusters at the leading edge of the FEL pulse. We have shown that the method is sensitive to the target composition. The accurate modeling of a target ionization in the focus volume [46] is complicated by significant shot-toshot fluctuations of almost all FEL pulse parameters such as pulse energy and pulse duration as well as spatial intensity distribution [1,2]. In our study we show the applicability of the THz-field streaking technique to temporally resolve the electron emission from extended targets under intense FEL radiation on time scales that are significantly shorter than the FEL pulse duration. This is a regime in which NIR (or attosecond) streaking cannot be applied, because the required NIR intensities would lead to additional ionization of the clusters. This can be avoided by using a $\mathrm{THz}$ pulse with a much lower intensity, but with a similar ponderomotive energy. We expect that our results will stimulate further development of the theory of the light-field streaking which considers the multielectron dynamics in highly excited systems. With continuous development of $\mathrm{THz}$ sources that are able to provide intense single or multicycle $\mathrm{THz}$ pulses [47], these types of experiments will significantly advance the ultrafast $\mathrm{x}$-ray physics at existing and emerging state-of-the-art user facilities [1-3] as well as in the laboratory [48].

\section{ACKNOWLEDGMENTS}

We acknowledge Thomas Möller and Thomas Fennel for fruitful discussions. The authors would like to thank 
the scientific and technical team of FLASH for their outstanding support. This work has been supported by the project Structural dynamics of biomolecular systems (No. CZ.02.1.01/0.0/0.0/15_003/0000447) from European
Regional Development Fund and by the project Advanced research using high intensity laser produced photons and particles (No. CZ.02.1.01/0.0/0.0/16_019/0000789) from European Regional Development Fund.
[1] J. Feldhaus, M. Krikunova, M. Meyer, T. Möller, R. Moshammer, A. Rudenko, T. Tschentscher, and J. Ullrich, J. Phys. B 46, 164002 (2013).

[2] E. A. Seddon, J. A. Clarke, D. J. Dunning, C. Masciovecchio, C. J. Milne, F. Parmigiani, D. Rugg, J. C. H. Spence, N. R. Thompson, K. Ueda et al., Rep. Prog. Phys. 80, 115901 (2017).

[3] L. Young, K. Ueda, M. Gühr, P. H. Bucksbaum, M. Simon, S. Mukamel, N. Rohringer, K. C. Prince, C. Masciovecchio, M. Meyer et al., J. Phys. B 51, 032003 (2018).

[4] A. A. Sorokin, S. V. Bobashev, T. Feigl, K. Tiedtke, H. Wabnitz, and M. Richter, Phys. Rev. Lett. 99, 213002 (2007).

[5] N. Rohringer, D. Ryan, R. A. London, M. Purvis, F. Albert, J. Dunn, J. D. Bozek, C. Bostedt, A. Graf, R. Hill et al., Nature (London) 481, 488 (2012).

[6] S. Shwartz, M. Fuchs, J. B. Hastings, Y. Inubushi, T. Ishikawa, T. Katayama, D. A. Reis, T. Sato, K. Tono, M. Yabashi et al., Phys. Rev. Lett. 112, 163901 (2014).

[7] A. Rudenko, L. Inhester, K. Hanasaki, X. Li, S. J. Robatjazi, B. Erk, R. Boll, K. Toyota, Y. Hao, O. Vendrell et al., Nature (London) 546, 129 (2017).

[8] L. Young, E. P. Kanter, B. Krässig, Y. Li, A. M. March, S. T. Pratt, R. Santra, S. H. Southworth, N. Rohringer, L. F. DiMauro et al., Nature (London) 466, 56 (2010).

[9] Y. Ovcharenko, V. Lyamayev, R. Katzy, M. Devetta, A. LaForge, P. OKeeffe, O. Plekan, P. Finetti, M. Di Fraia, M. Mudrich et al., Phys. Rev. Lett. 112, 073401 (2014).

[10] K. Nagaya, Iablonskyi, Golubev, K. Matsunami, H. Fukuzawa, K. Motomura, T. Nishiyama, T. Sakai, T. Tachibana, S. Mondal et al., Nat. Commun. 7, 13477 (2016).

[11] Y. H. Jiang, A. Rudenko, O. Herrwerth, L. Foucar, M. Kurka, K. U. Kühnel, M. Lezius, M. F. Kling, J. van Tilborg, A. Belkacem et al., Phys. Rev. Lett. 105, 263002 (2010).

[12] M. Krikunova, T. Maltezopoulos, P. Wessels, M. Schlie, A. Azima, T. Gaumnitz, T. Gebert, M. Wieland, and M. Drescher, Phys. Rev. A 86, 043430 (2012).

[13] C. Kupitz, S. Basu, I. Grotjohann, R. Fromme, N. A. Zatsepin, K. N. Rendek, M. S. Hunter, R. L. Shoeman, T. A. White, D. Wang et al., Nature (London) 513, 261 (2014).

[14] R. Neutze, R. Wouts, D. van der Spoel, E. Weckert, and J. Hajdu, Nature (London) 406, 752 (2000).

[15] H. N. Chapman, C. Caleman, and N. Timneanu, Philos. Trans. R. Soc. B 369, 20130313 (2014).

[16] H. O. Jönsson, C. Östlin, H. N. Chapman, S. J. Aplin, N. Timneanu, and C. Caleman, High Energy Density Phys. 26, 93 (2018).

[17] M. Krikunova, M. Adolph, T. Gorkhover, D. Rupp, S. Schorb, C. Bostedt, S. Roling, B. Siemer, R. Mitzner, H. Zacharias et al., J. Phys. B 45, 105101 (2012).

[18] A. D. Rath, N. Timneanu, F. R. N. C. Maia, J. Bielecki, H. Fleckenstein, B. Iwan, M. Svenda, D. Hasse, G. Carlsson, D. Westphal et al., Opt. Express 22, 28914 (2014).
[19] B. Schütte, T. Oelze, M. Krikunova, M. Arbeiter, T. Fennel, M. J. J. Vrakking, and A. Rouzée, New J. Phys. 17, 033043 (2015).

[20] B. Schütte, T. Oelze, M. Krikunova, M. Arbeiter, T. Fennel, M. J. J. Vrakking, and A. Rouzée, J. Phys. B 48, 185101 (2015).

[21] T. Gorkhover, S. Schorb, R. Coffee, M. Adolph, L. Foucar, D. Rupp, A. Aquila, J. Bozek, S. W. Epp, B. Erk et al., Nat. Photon. 10, 93 (2016).

[22] L. Flückiger, D. Rupp, M. Adolph, T. Gorkhover, M. Krikunova, M. Müller, T. Oelze, Y. Ovcharenko, M. Sauppe, S. Schorb et al., New J. Phys. 18, 043017 (2016).

[23] Y. Kumagai, H. Fukuzawa, K. Motomura, D. Iablonskyi, K. Nagaya, S. Wada, Y. Ito, T. Takanashi, Y. Sakakibara, D. You et al., Phys. Rev. X 8, 031034 (2018).

[24] M. Drescher, M. Hentschel, R. Kienberger, M. Uiberacker, V. Yakovlev, A. Scrinzi, T. Westerwalbesloh, U. Kleineberg, U. Heinzmann, and F. Krausz, Nature (London) 419, 803 (2002).

[25] R. Kienberger, E. Gouliemakis, M. Ueberacker, A. Baltuska, V. Yakovlev, F. Bammer, A. Scrinzi, T. Westerwalbesloh, U. Kleineberg, U. Heinzmann et al., Nature (London) 427, 817 (2004).

[26] J. Itatani, F. Quéré, G. L. Yudin, and P. B. Corkum, Laser Phys. 14, 344 (2004).

[27] M. Schultze, M. Fieß, N. Karpowicz, J. Gagnon, M. Korbman, M. Hofstetter, S. Neppl, A. L. Cavalieri, Y. Komninos, T. Mercouris et al., Science 328, 1658 (2010).

[28] A. L. Cavalieri, N. Müller, T. Uphues, V. S. Yakovlev, A. Baltuska, B. Horvath, B. Schmidt, L. Blümel, R. Holzwarth, S. Hendel et al., Nature (London) 449, 1029 (2007).

[29] L. Seiffert, Q. Liu, S. Zherebtsov, A. Trabattoni, P. Rupp, M. C. Castrovilli, M. Galli, F. Süssmann, K. Wintersperger, J. Stierle et al., Nat. Phys. 13, 766 (2017).

[30] M. Arbeiter and T. Fennel, Phys. Rev. A 82, 013201 (2010).

[31] C. Bostedt, H. Thomas, M. Hoener, E. Eremina, T. Fennel, K.H. Meiwes-Broer, H. Wabnitz, M. Kuhlmann, E. Plönjes, K. Tiedtke et al., Phys. Rev. Lett. 100, 133401 (2008).

[32] C. Bostedt, H. Thomas, M. Hoener, T. Möller, U. Saalmann, I. Georgescu, C. Gnodtke, and R. J.-M., New J. Phys. 12, 083004 (2010).

[33] U. Frühling, M. Wieland, M. Gensch, T. Gebert, B. Schütte, M. Krikunova, R. Kalms, F. Budzyn, O. Grimm, J. Rossbach et al., Nat. Photon. 3, 523 (2009).

[34] B. Schütte, S. Bauch, U. Frühling, M. Wieland, M. Gensch, E. Plönjes, T. Gaumnitz, A. Azima, M. Bonitz, and M. Drescher, Phys. Rev. Lett. 108, 253003 (2012).

[35] U. Becker and D. A. Shirley, VUV and Soft X-ray Photoionization (Springer, New York, 1996).

[36] M. Arbeiter and T. Fennel, New J. Phys. 13, 053022 (2011).

[37] M. Gensch, L. Bittner, A. Chesnov, H. Delsim-Hashemi, M. Drescher, B. Faatz, J. Feldhaus, U. Fruehling, G. Geloni, C. Gerth et al., Infrared Phys. Technol. 51, 423 (2008).

[38] R. W. Boyd, J. Opt. Soc. Am. 70, 877 (1980). 
[39] T. Oelze, B. Schütte, M. Müller, J. P. Müller, M. Wieland, U. Frühling, M. Drescher, A. Al-Shemmary, T. Golz, N. Stojanivic et al., Sci. Rep. 7, 40736 (2016).

[40] O. F. Hagena and W. Obert, J. Chem. Phys. 56, 1793 (1972).

[41] M. Hoener, C. Bostedt, H. Thomas, L. Landt, E. Eremina, H. Wabnitz, T. Laarmann, R. Treusch, A. R. B. de Castro, and T. Möller, J. Phys. B 41, 181001 (2008).

[42] L. Schroedter, M. Müller, A. Kickermann, A. Przystawik, S. Toleikis, M. Adolph, L. Flückiger, T. Gorkhover, L. Nösel, M. Krikunova et al., Phys. Rev. Lett. 112, 183401 (2014).
[43] M. Tchaplyguine, M. Lundwall, M. Gisselbrecht, G. Ohrwall, R. Feifel, S. Sorensen, S. Svensson, N. Martensson, and O. Bjorneholm, Phys. Rev. A 69, 031201(R) (2004).

[44] U. Saalmann, J. Phys. B 43, 194012 (2010).

[45] C. Gnodtke, U. Saalmann, and J.-M. Rost, New J. Phys. 13, 013028 (2011)

[46] M. G. Makris, P. Lambropoulos, and A. Mihelic, Phys. Rev. Lett. 102, 033002 (2009).

[47] N. Stojanovic and M. Drescher, J. Phys. B 46, 192001 (2013).

[48] B. Schütte, M. Arbeiter, T. Fennel, M. J. J. Vrakking, and A. Rouzée, Phys. Rev. Lett. 112, 073003 (2014). 